UAB-FT-322

September 1993

\title{
LOOP ACTION FOR LATTICE U(1) GAUGE THEORY
}

\author{
J. M. Aroca \\ Departament de Matemàtiques, \\ Universitat Politècnica de Catalunya, \\ Barcelona, Spain \\ and \\ H. Fort \\ Grup de Física Teòrica \\ and \\ Institut de Física d'Altes Energies \\ Universitat Autònoma de Barcelona \\ 08193 Bellaterra (Barcelona) Spain
} Escola Tècnica Superior d'Enginyers de Telecomunicació, E-08034

\begin{abstract}
It is showed that the very recently introduced Lagrangian loop formulation of the lattice Maxwell theory is equivalent to the Villain form in $2+1$ dimensions. A transparent description of the classical loop action is given in pure geometrical terms for the $2+1$ and $3+1$ dimensional cases.
\end{abstract}


The loop formalism was introduced some time ago as a general analytical Hamiltonian approach based on the properties of the group of loops [1]. It provides a common framework for quantum gauge theories - it works for several models, as Maxwell theory [2], Chern-Simons theory [3], etc - and quantum gravity [4. Furthermore, it works for Yang-Mills theories on a lattice [5] and recently has been extended in such a way to take account of dynamical fermions [6]. Very recently [7] a loop action for the U(1) gauge theory has been built which lattice version leads to the Kogut-Susskind Hamiltonian. A Lagrangian formulation in terms of loops is interesting for multiple reasons. First, it offers the possibility of knit together the intrinsic advantages of the nonlocal loop description (the non-redundancy of gauge degrees of freedom and its geometrical transparency) and the computational power of numerical simulations. This provides a a useful complement to the analytical loop studies. The classical action may be also relevant to perform semiclassical approximations. In this paper we show that the proposed action for the $\mathrm{D}=2+1$ dimensional case leads after a duality transformation to the discrete Gaussian model form. It is known that the same happens for the Villain form. Thus, for $\mathrm{D}=2+1$, the minimal description provided by the loops actually corresponds to that of Villain form instead of Wilson cosinus form. For $\mathrm{D}=3+1$ the loop action and Villain form give a similar description but the connection between them is more subtle.

According to reference [7] the continuum Euclidean loop action for the abelian theory in $D=d+1$ space-time dimensions is given by

$$
S=\frac{g^{2}}{2} \int d t d^{d} x\left\{-\dot{X}_{C}^{i}(\mathbf{x}) \frac{1}{\Delta} \dot{X}_{C}^{i}(\mathbf{x})+X_{C}^{i}(\mathbf{x}) X_{C}^{i}(\mathbf{x})\right\}
$$

where $X_{C}^{i}(\mathbf{x})$ is the loop current $\oint_{C} d y^{i} \delta^{3}(\mathbf{x}-\mathbf{y})$ and $\Delta$ is the three-dimensional Laplace operator. The time derivative of $X_{C}^{i}(\mathbf{x})$ can be written as $\lim _{d t \rightarrow 0}$ $\frac{1}{d t} \oint_{C_{t+d t} \bar{C}_{t}} d y^{i} \delta^{3}(\mathbf{x}-\mathbf{y})$, where $\bar{C}_{t}$ denotes the loop $C_{t}$ traversed in the opposite direction.

In order to formulate (11) on a lattice we represent the continuum surface spanned by the loop $C$ as a set of spatial loops $C_{t}$ at different times $t=$ $0,1, \ldots, T$. We also replace the derivatives by finite difference operators and get

$$
S=\frac{g^{2}}{2} \sum_{t} \sum_{s}\left\{-X_{C_{t+1} \bar{C}_{t}}^{i}(s) \frac{1}{\Delta} X_{C_{t+1} \bar{C}_{t}}^{i}(s)+X_{C_{t}}^{i}(s) X_{C_{t}}^{i}(s)\right\}
$$


where $X_{C_{t}}^{i}(s)$ is an integer which counts the number of times that the loop $C_{t}$ traverses the link $(s, \hat{i})$.

We shall consider first the $\mathrm{D}=2+1$ dimensional case. The loops $C_{t}$ lie on a plane and have a unique decomposition in plaquettes $C_{t}=\prod_{p} p^{n_{p}}$, where $n_{p}$ is the plaquette multiplicity, i.e. the number of times that plaquette $p$ is contained in loop $C$. The first term in (2) is then

$$
\sum_{s}-X_{D}^{i}(s) \frac{1}{\Delta} X_{D}^{i}(s)=\sum_{p, p^{\prime}} \sum_{s}-n_{p} n_{p^{\prime}} X_{p}^{i}(s) \frac{1}{\Delta} X_{p^{\prime}}^{i}(s)=\sum_{p, p^{\prime}} n_{p} n_{p^{\prime}} l\left(p, p^{\prime}\right)
$$

where $D$ denotes the loop $C_{t+1} \bar{C}_{t}, l\left(p, p^{\prime}\right)$ is the interaction between two plaquettes and is given by

$$
l\left(p, p^{\prime}\right) \equiv \sum_{s}-X_{p}^{i}(s) \frac{1}{\Delta} X_{p^{\prime}}^{i}(s)=\sum_{s, s^{\prime}} X_{p}^{i}(s) G\left(s, s^{\prime}\right) X_{p^{\prime}}^{i}\left(s^{\prime}\right)
$$

with

$$
G\left(s, s^{\prime}\right)=\int_{0}^{2 \pi} \frac{d^{2} q}{(2 \pi)^{2}} \frac{e^{i q\left(s-s^{\prime}\right)}}{2\left(2-\cos q_{1}-\cos q_{2}\right)}=\delta_{s, s^{\prime}} .
$$

That gives

$$
\sum_{s}-X_{D}^{i}(s) \frac{1}{\Delta} X_{D}^{i}(s)=\sum_{p, p^{\prime}} n_{p} n_{p^{\prime}} \delta_{p, p^{\prime}}=\sum_{p} n_{p}^{2}=A_{2}(D)
$$

where $A_{2}(D)$ actually denotes the quadratic area, i.e. the sum of the squares of the constitutive plaquette multiplicities, of the surface $\mathcal{S}$ enclosed by the loop $D$.

The second term on the action is

$$
\sum_{s} X_{C}^{i}(s) X_{C}^{i}(s)=\Lambda(C)
$$

where $\Lambda(C)$ denotes the quadratic length -the sum of squares of link multiplicities- of the loop $C$.

We arrive then to the following geometric expression for the lattice action:

$$
S=\frac{g^{2}}{2} \sum_{t}\left\{A_{2}\left(C_{t+1} \bar{C}_{t}\right)+\Lambda\left(C_{t}\right)\right\}
$$


Now, instead of expressing the loops as an integer field defined on the links of the lattice, we express them through the surface they enclose by giving an integer field defined on the spatial plaquettes. By duality this field is defined on the sites $(\tilde{x}, \tilde{y}, t)$ dual to these spatial plaquettes so the configurations are given by integer fields $k(\tilde{x}, \tilde{y}, t)$. The geometric terms in the action are expressed then as

$$
\Lambda\left(C_{t}\right)=\sum_{\tilde{x}, \tilde{y}, t}\left\{(k(\tilde{x}+1, \tilde{y}, t)-k(\tilde{x}, \tilde{y}, t))^{2}+(k(\tilde{x}, \tilde{y}+1, t)-k(\tilde{x}, \tilde{y}, t))^{2}\right\}
$$

and

$$
A_{2}\left(C_{t+1} \bar{C}_{t}\right)=\sum_{\tilde{x}, \tilde{y}, t}(k(\tilde{x}, \tilde{y}, t+1)-k(\tilde{x}, \tilde{y}, t))^{2} .
$$

Collecting these results, the action reads finally

$$
S=\frac{g^{2}}{2} \sum_{s, \mu}(k(s+\hat{\mu})-k(s))^{2}
$$

which is the action of the integer Gaussian spin model. In this expression the covariance hidden in (2) is manifest.

On the other hand, the duality of the Villain form of the $\mathrm{U}(1)$ gauge theory to discrete Gaussian models - discrete Gaussian spin model for $\mathrm{D}=3$, discrete Gaussian gauge model for $\mathrm{D}=4$ - was already established [9], [10]. Thus, for $\mathrm{D}=3$, we have found that the minimal description that provides the loop action (2) is equivalent to the Villain form since that both lead by duality to (12).

With the aim to explore this connection between loops and Villain form for an arbitrary dimension $\mathrm{D}$ we shall resort to the language of forms. Working in this general framework we will show that we recover the previous equivalence for $\mathrm{D}=2+1$ and we will analyze in detail the relation between both theories at $\mathrm{D}=3+1$.

A p-form is a function defined on the p-cells of the lattice $(\mathrm{p}=0$ sites, $\mathrm{p}=1$ links, $\mathrm{p}=2$ cubes, etc.) over an abelian group which shall be $\mathbf{R}, \mathbf{Z}$, or $\mathrm{U}(1)=$ reals module $2 \pi$. Integer forms can be considered geometrical objects on the lattice. For instance, a 1-form is a path and the integer value on a link is the number of times that the path traverses this link. $\nabla$ is the 
co-border operator which maps $\mathrm{p}$-forms onto $(\mathrm{p}+1)$-forms. It is the gradient operator when acting on scalar functions (0-forms) and it is the rotational on vector functions (1-forms). We shall consider the scalar product of $\mathrm{p}$ forms defined $\langle\alpha \mid \beta\rangle=\sum_{c_{p}} \alpha(c) \beta(c)$ where the sum runs over the p-cells of the lattice. Under this product the $\nabla$ operator is adjoint to the border operator $\partial$ which maps p-forms onto (p-1)-forms and which corresponds to minus times the usual divergence operator. The operator $\square=\nabla \partial+\partial \nabla$ is called the Laplacian and differs only by a minus sign of the current Laplacian $\Delta_{\mu} \Delta_{\mu}$. We shall use also the duality transformation which maps biyectively p-forms over (D-p)-forms. Under duality the border and co-border operators interchange.

In the loop theory exposed before, physical configurations correspond to distributions of spatial loops at different times. These are spatial closed integer 1 -forms. Then we consider only forms $c$ which are 0 for temporal links and closed $(\partial c=0)$. The border and co-border operators can be restricted to the spatial sections ( $\mathrm{t}=$ constant) and we denote the spatial Laplacian as $\Delta$. The loop action (2) can be expressed as

$$
S=\frac{g^{2}}{2} \sum_{t}\left(<c_{t+1} \bar{c}_{t}, \frac{1}{\Delta} c_{t+1} \bar{c}_{t}>+<c_{t}, c_{t}>\right)=\frac{g^{2}}{2}<c, \frac{\square}{\Delta} c>
$$

and the path integral

$$
Z=\sum_{c} \exp \left(-\frac{g^{2}}{2}<c, \frac{\square}{\Delta} c>\right)
$$

where $\mathrm{c}$ denotes a spatial loop i.e. $\mathrm{c}$ belongs to a $\mathrm{t}=$ constant slice.

It is possible to solve the constraint $\partial c=0$ by taking $c=\partial s$ where $\mathrm{s}$ are integer spatial 2-forms.

$$
Z=\sum_{s}^{\prime} \exp \left(-\frac{g^{2}}{2}<\partial s, \frac{\square}{\Delta} \partial s>\right)
$$

The prime on the sum indicates that we must restrict the $s$ 's over which we sum since we have introduced the symmetry $s \rightarrow s+\partial v$ for arbitrary 3 -forms $v$ (in other words, there are infinite surfaces with the same loop as 
border). This restriction is the usual gauge-fixing problem and is solved more simply if we make a duality transformation in the spatial sections. In terms of the spatial (D-3)-forms $* s(14)$ is written as

$$
Z=\sum_{* s} \exp \left(-\frac{g^{2}}{2}<\nabla * s, \frac{\square}{\Delta} \nabla * s>\right)
$$

For $\mathrm{D}=2+1$ the dual of $* s$ are 0 -forms $* s$. We do not need to fix the gauge since a spatial loop in the border of a unique surface.

If we use the Poisson sumation formula $\sum_{j} f(j)=\sum_{k} \int_{-\infty}^{\infty} d \phi f(\phi) e^{2 \pi i \phi k}$ we can substitute the integer $* s$ variables by integer 0 -forms $* m$ and real 0 -forms $\phi$, and performing the Gaussian integration over $\phi$ we get

$$
Z=\sum_{m} \exp \left(-\frac{2 \pi^{2}}{g^{2}}<* m, \frac{1}{\square} * m>\right)
$$

The above expression is a sum over point monopoles which interact through a Coulomb potential. Its form is equal to the one obtained from the Villain form of the $U(1)$ theory. Those monopoles are defined in the centers of the spatial plaquettes and these configurations are in correspondence one-to-one with scalar forms on the three-dimensional lattice. In this case, as we have seen before, the loop theory describes exactly the topological excitation of the Villain theory.

For the $\mathrm{D}=3+1$ case the dual of the $s$ 's in (14) are spatial 1-forms $* s$. To fix $* s$ we impose $* s(l)=0$ for every link $l \in T$, T being a maximal spatial tree. This selects only one $* s$ among all $* s$ 's with the same value of $\nabla * s$. If we use the Poisson sumation formula we can substitute the integer $* s$ variables on the complementary of $T, \bar{T}$ by integer 1 -forms $* m$ and real 1 -forms $\phi$. Once again the $\phi$ integration gives

$$
\begin{aligned}
& Z=\text { constant } \quad \sum_{* m} \exp \left(-\frac{2 \pi^{2}}{g^{2}}<* m, \frac{1}{\square} * m>\right) \\
& (\partial * m=0)
\end{aligned}
$$

i.e. a sum over spatial closed loops which interact through a Coulomb potential. The Villain form of the $U(1)$ gauge theory leads to the same expression but the monopole loops are not restricted to lie at $\mathrm{t}=$ constant. 
Thus, the loop theory is not fully equivalent to the Villain $\mathrm{U}(1)$ theory at $\mathrm{D}=4$.

We can deepen our insight on the relation between the loop and the Villain theory following the opposite way: starting from Villain form and then repeating the previous treatment in terms of p-forms. The path integral for the Villain $\mathrm{U}(1)$ theory is

$$
Z=\int(d \theta) \sum_{j} \exp \left(-\frac{\beta}{2}\|\nabla \theta-2 \pi j\|^{2}\right)
$$

where $\theta$ is a real periodic 1 -form, that is, a real number $\theta_{l} \in[0,2 \pi]$ defined in each link of the lattice. $j$ are integer 2 -forms, defined at the lattice plaquettes, and $\|.\|^{2}=<., .>$. Using the Poisson sumation formula and integrating first in the continuum $\phi$ variables and then over the compact $\theta$, we finally get

$$
\begin{aligned}
& Z=(2 \pi \beta)^{-N_{p} / 2} \quad \sum_{s} \exp \left(-\frac{1}{2 \beta}<s, s>\right) \propto \sum_{s} \exp \left(-\frac{1}{2 \beta} A_{2}(\mathcal{S})\right) \\
& (\partial s=0)
\end{aligned}
$$

where $N_{p}$ in the number of plaquettes of the lattice. We can now analyze the geometrical meaning of this equation. $s$ are integer 2-forms, that is they are defined over bidimensional surfaces on the lattice. The condition $\partial s=0$ means to sum only over closed surfaces, the action $\langle s, s\rangle$ is proportional to the quadratic area of the surface. Selecting one direction of the lattice as a 'time' direction one can express $\langle s, s>$ as (see Appendix)

$$
<s, s>=\sum_{t}\left(<c_{t}, c_{t}>+<c_{t+1} \bar{c}_{t}, \frac{1}{\Delta} c_{t+1} \bar{c}_{t}>+<\nabla \mathbf{s}_{t}, \frac{1}{\Delta} \nabla \mathbf{s}_{t}>\right)
$$

where $\mathbf{s}_{t}$ represents a pure spatial 2 -form. The first two terms in (20) correspond exactly to the loop action (12). If we work at $\mathrm{D}=2+1$ we have only one solution to $\partial \mathbf{s}_{t}=c_{t+1} \bar{c}_{t}$. In this case the spatial co-border of a 2form is 0 , then the third term in (20) vanishes and we obtain the loop theory (12). 
For $\mathrm{D}=3+1$ the loop action can not take account of the term $<\nabla \mathbf{s}_{t}, \frac{1}{\square} \nabla \mathbf{s}_{t}>$. The Lagrangian lattice loop description seems to miss some degrees of freedom present in the gauge $\mathrm{U}(1)$ theory (the general monopole loops which are not contained in the $t=$ constant hypersurfaces). As it was previously mentioned it has been shown that the loop action leads to the Kogut-Susskind Hamiltonian so for continuous time both descriptions are equivalent.

It seems interesting to have a Lagrangian lattice loop description to continue exploring some interesting points. For example, the pure $3+1 \mathrm{U}(1)$ gauge theory was studied by means of Hamiltonian analytical method in the loop representations [8]. In this reference we found a continuous phase transition for this model instead of the first order measured by means of standard Monte Carlo simulations. A possible explanation for this difference relies on the fact that we have used a cluster method which implies a formally infinite lattice which is free from the spurious topological effects occurring in finite lattices with periodic boundary conditions [12] [13]. This periodicity leads, on the confining side, to large monopole loops which wrap around the lattice. Those loops clearly have a fairly large action associated with them, so a jump is observed when they disappear at deconfining transition. This jump no longer survives in the infinite volume limit. The effect of this wrapping loops in driving the transition is crucial. Recently, Stack and Wensley [14 have calculated the heavy quark potential from the magnetic current due the monopoles. They have resolved the magnetic current into large loops which wrap around the lattice and simple loops which do not. They found that the long range part of the heavy quark potential can be calculated solely from the large, wrapping loops of magnetic current.

The loop's method seems to be in a good position because the finite lattice is not one of its intrinsic features. The development of different Lagrangian computational techniques for studying the $\mathrm{D}=4 \mathrm{U}(1)$ model in order to complement our previous Hamiltonian results is one of our present aims. Also, we are interested in extending the loop's action in such a way to include matter fields.

We wish to thank, D.Armand-Ugon, R.Gambini, M.Grady and L.Setaro for useful discussions and comments.

\section{APPENDIX}

Selecting a direction on the lattice which we call time and consider the 
division of the lattice in space sections of dimension D-1. Any surface 2-form $s$ can be written $s=\sum_{t}\left(\mathbf{s}_{t}+\tilde{s}_{t}\right)$ where $\mathbf{s}$ is purely spatial and $\tilde{s}$ is temporal (its plaquettes have one link in the $t$ direction joining the space sections $t$ and $t+1)$. The $t$ subindex correspond to each spatial slice of the lattice. If we consider the borders of these surfaces we have that they are always spatial 1-forms (loops) in the slice $t$ since the temporal parts of $\partial \tilde{s}_{t}$ cancel. $\partial \tilde{s}_{t}$ has two spatial components in the sections $t$ and $t+1,\left(\partial \tilde{s}_{t}\right)_{\text {inf }}$ and $\left(\partial \tilde{s}_{t}\right)_{\text {sup }}$. We define $c_{t}=\left(\partial \tilde{s}_{t}\right)_{\text {sup }}$. Then $\left(\partial \tilde{s}_{t}\right)_{\text {inf }}=-c_{t}$. Imposing $s$ to be closed we have $\partial \mathbf{s}_{t}=c_{t+1}-c_{t}$. We note also that if $p$ is a plaquette defined by a spatial link $l$ and a temporal link, then the integers $\tilde{s}_{t}(p)$ and $c_{t}(l)$ coincide. The quadratic area is then

$$
\begin{gathered}
<s, s>=\sum_{t}\left(<\tilde{s}_{t}, \tilde{s}_{t}>+<\mathbf{s}_{t}, \mathbf{s}_{t}>\right)= \\
\sum_{t}\left(<\tilde{s}_{t}, \tilde{s}_{t}>+<\partial \mathbf{s}_{t}, \frac{1}{\Delta} \partial \mathbf{s}_{t}>+<\nabla \mathbf{s}_{t}, \frac{1}{\Delta} \nabla \mathbf{s}_{t}>\right)= \\
\sum_{t}\left(<c_{t}, c_{t}>+<c_{t+1} \bar{c}_{t}, \frac{1}{\Delta} c_{t+1} \bar{c}_{t}>+<\nabla \mathbf{s}_{t}, \frac{1}{\Delta} \nabla \mathbf{s}_{t}>\right)
\end{gathered}
$$

In the second equality we have inserted in the second term $\square \square^{-1}$ and used the adjoint relation between border and coborder operators. In this expression the operators belong to the spatial hypersurface, so they 'live' in dimension D-1. In the path integral the sum over $s$ is replaced for a sum over the distributions of spatial loops $c_{t}$ and spatial surfaces $s_{t}$ such that $\partial \mathbf{s}_{t}=c_{t+1} \bar{c}_{t}$. 


\section{References}

[1] R.Gambini and A.Trias, Phys.Rev.D 23, 553 (1981).

[2] R.Gambini and A.Trias, Phys.Rev.D 22, 1380 (1980).

[3] M.Li, Nuo.Cim. B105, 1113 (1990)

[4] A.Ashtekar, V.Husain, C.Roveli, J.Samuel and L.Smolin, Class.Quant.Grav. 6 L185 (1989).

[5] R.Gambini and A.Trias, Nuc. Phys. B278, 436 (1986).

[6] H.Fort and R.Gambini, Phys.Rev.D 44, 1257 (1991).

[7] D.Armand Ugon, R.Gambini, J.Griego and L.Setaro, Preprint July, 1993.

[8] J.M.Aroca and H.Fort, Phys.Lett.B299, 305 (1993).

[9] T. Banks, R. Myerson and J.B. Kogut, Nucl. Phys. B129 493 (1977).

[10] R.Savit, Rev.Mod.Phys.52 453 (1980).

[11] A.M. Polyakov, Nuc. Phys. B120 429 (1977).

[12] M. Grady, Nucl. Phys. B365 699 (1991).

[13] R.Gupta, M.Novotny and R.Cordery, Phys.Lett. B172

[14] J.Stack and R.Wensley, Preprint ILL-(TH)-93 \#15 July, 1993. 